\title{
Acercamiento teórico para la integración de los conceptos de Resiliencia en los indicadores de Sostenibilidad Urbana.
}

A theoretical approach for integrating resiliency concepts to urban sustainability indicators.

Irina Tumini

\section{Filiación}

Universidad del Bío Bío, Departamento de Planificación y Diseño Urbano

E mail: irina.tumini@gmail.com

Primera versión recibida en: 18 de abril, 2016

Última versión recibida en: 28 de junio, 2016

\section{Resumen}

El Marco de Sendai para la Reducción del Riesgo de Desastre 2015-2030 propone la resiliencia y la gestión integral del riesgo como requisito indispensable para el desarrollo sostenible. El artículo presenta una discusión sobre la aplicación en el entorno construido de los conceptos de sostenibilidad y resiliencia. Aunque se reconoce un cierto entendimiento entre sostenibilidad y resiliencia, es incierta la identificación de las sinergias entre ambos conceptos. El objetivo del trabajo es proponer, a través de un acercamiento teórico un marco metodológico para la incorporación de los atributos de la resiliencia en los instrumentos de planificación sostenible. En el documento se subrayan los elementos que requieren de un cambio de enfoque para la adaptación a las nuevas exigencias, así como las sinergias claves que deberían potenciarse para el éxito del entorno urbano.

\section{Palabras Clave}

Resiliencia urbana; ciudades sostenibles; planificación urbana

\begin{abstract}
The Sendai framework for the Disaster Risk Reduction (2015-2030) recognizes that the resilience and the disaster risk reduction of human systems is a requirement for sustainable development. According to this, the paper presents a discussion about the two concepts of Sustainability and Resilience applied at the urban environment. Despite scholars recognize a general agreement between Sustainability and Resilience, there exist several uncertainties in identifying synergies between them. The aim of this work is proposing, through a theoretical approach, a methodological framework for urban planning tools adapting from the sustainable design to resilience requirements. In addition, the document highlights the need to re-focus some elements in order to cites adaptation, as well as synergies that should be strengthened because keys to change the urban environment.
\end{abstract}

\section{Key words}

Urban resilience; sustainable cities; urban planning 


\section{Sumario}

1 Introducción

1.1 Indicadores de sostenibilidad urbana CAT-MED

1.2 El concepto de Resiliencia aplicado al entorno urbano

2 Análisis de los indicadores

2.1 Territorio y configuración de la ciudad

2.2 Movilidad y Transporte

2.3 Gestión de Recursos Naturales

2.4 Cohesión social y económica

2.5 Indicadores Socio-Económicos Complementarios

3 Resultados de la comparativa entre indicadores de sostenibilidad y de resiliencia

4 Discusiones

5 Conclusiones

Referencias

\section{I ntroducción}

En las últimas décadas el concepto de sostenibilidad se ha consolidado como el hilo conductor de científicos, ambientalistas y todas aquellas personas preocupadas por el complejo y frágil sistema que habitamos. El debate sobre la sostenibilidad ha involucrado todos los sectores, incluida la planificación urbana, materializándose en una nutrida colección de políticas, normas, guías y herramientas con el propósito de orientar el desarrollo urbano hacía modelos más eficientes.

En relación con la aplicación de los principios de la sostenibilidad en los espacios urbanos se han generado numerosas iniciativas orientadas su medición y cuantificación a múltiples escalas. La definición y monitorización de indicadores de sostenibilidad ha dado la posibilidad a organizaciones y autoridades gubernamentales de observar las tendencias, trazar lineamientos y políticas y en general, transformar un concepto abstracto en objetivos cuantificables. Sin embargo, estos indicadores han recibido numerosas críticas debido a la dificultad en su interpretación, así como, a la falta de conexión entre los parámetros medidos y los objetivos esperados (Milman y Short 2008).

En la actualidad existen críticas respecto a los modelos de sostenibilidad urbana puesto que son insuficientes para preparar a las ciudades frente a los desastres naturales y el cambio climático. En los últimos diez años los desastres naturales han sido responsables de la muerte de 700.000 personas, más de 1,4 millones han sufrido heridas y alrededor de 23 millones se han quedado sin hogar (UNISDR 2015). En este último decenio, las pérdidas debidas a los desastres alcanzan los 200 billones de dólares (Word Bank 2015), un valor en constante aumento debido al crecimiento demográfico, a la rápida urbanización y al calentamiento global.

De acuerdo al Marco de Sendai para la Reducción del Riesgo de Desastres 2015-2030 (Marco Sendai), la prevención del riesgo de desastre es una inversión rentable para la reducción de las 
pérdidas futuras y un elemento indispensable para alcanzar los objetivos del desarrollo sostenible. Este acuerdo internacional remarca la necesidad de examinar las experiencias, estrategias, acciones y políticas de reducción del riesgo y capitalizar el aprendizaje para la definición de modalidades de cooperación entre los estados. En sintonía con el Marco de Acción de Hyogo para 2005-2015: Aumento de la Resiliencia de las Naciones y las Comunidades ante los Desastres este nuevo acuerdo hace énfasis en la necesidad de reforzar la "resiliencia" de las comunidades a todos los niveles, asegurando los medios necesarios para lograrlo. En él aparece también el concepto de "sostenibilidad" como objetivo para el crecimiento, orientado hacia la eficiencia en el uso de los recursos y a la creación de sistemas de asistencia financiera estable que permitan el desarrollo equitativo de la sociedad (UNISDR 2015, 2005).

Se evidencia así una estrecha relación entre los dos conceptos, interpretándose la mejora de la resiliencia como indispensable para el desarrollo sostenible de las comunidades. El Banco Mundial, en la guía para la recuperación resiliente, subraya que en la reconstrucción postdesastre uno de los objetivos principales es "trasladar las ganancias de la recuperación resiliente en el desarrollo sostenible" (GFDRR 2015, p. 10). Para que eso sea posible, las políticas, planes, prácticas y estrategias deben coordinarse con las agendas de desarrollo sostenible, la eficiencia en el uso de los recursos, la protección de los ecosistemas, la seguridad de las comunidades y la prevención del riesgo.

La visión del riesgo y del desastre como oportunidad para mejorar el entorno y promover el desarrollo sostenible está reclutando cada vez más seguidores cautivados por la atractiva posibilidad de valorizar las sinergias entre ambos conceptos. Sin embargo, la falta de referencias metodológicas y experiencias en el tema generan incertidumbres entre los científicos y los técnicos en su aplicación práctica en el entorno urbano. Además, el fracaso del Summit Rio+20 (2012) ha puesto en evidencia los desacuerdos entre los Países en cuanto a las acciones relacionadas con el Cambio Climático y el desarrollo sostenible (McCormick et al. 2013; Benson y Craig 2014).

Varios autores reconocen como principal limitación de la sostenibilidad el enfoque de estado estacionario, gracias al cual los sistemas, ecológicos y sociales, pueden subsistir en equilibrio con el entorno. En esta definición queda implícito el concepto de estabilidad de las condiciones iniciales y que, a través de la organización eficiente de los recursos disponibles, los sistemas antrópicos podrán subsistir de generación en generación. Sin embargo, los sistemas sostenibles no contemplan las crisis, ni los cambios repentinos e inesperados. Al ser menos vulnerables, los sistemas sostenibles pueden evitar el colapso inminente, manteniéndose en el borde del precipicio a la espera del próximo evento (Ahern 2011; Cascio 2009).

Por otro lado, el estudio de la resiliencia acepta el cambio como hecho inevitable y en algunos casos imprevisible, enfocándose en la necesidad de los sistemas de ser capaces de enfrentar las crisis, absorber las perturbaciones y reorganizarse (Walker et al. 2004). Los sistemas resilientes usan un enfoque de estabilidad no-lineal para comprender, explicar y diagnosticar los procesos locales de desarrollo (Walker et al. 2004).

Este trabajo nace de la necesidad de esclarecer las diferencias entre el enfoque sostenible y el resiliente, definiendo un marco teórico para organizar la integración de los principios de Resiliencia en las políticas e instrumentos de gobierno del territorio enfocados a la Sostenibilidad Urbana. A partir de los indicadores propuestos por el modelo urbano sostenible CAT-MED (2012) se evalúa la contribución de cada aspecto en la generación de entornos construidos más resilientes frente a los desastres naturales. La discusión teórica se organiza alrededor de la revisión de los parámetros de evaluación, ordenando los puntos clave de los dos enfoques, comparando diferencias y similitudes. En las conclusiones se hace énfasis en 
aquellos aspectos complementarios y en la necesidad de desarrollar sinergias entre los dos acercamientos para facilitar la transición de la ciudad a los nuevos retos de la sociedad.

\section{Marco Teórico}

Los gobiernos y autoridades cada vez se apoyan más en el uso de indicadores para la evaluación y monitoreo de los avances en materia de desarrollo sostenible. La proliferación del uso de sistemas medición se debe en primer lugar a la necesidad de sustentar de forma racional, eficiente y responsable la toma de decisiones. En segundo, a la capacidad que tienen dichos indicadores para medir las tendencias en la aplicación de políticas o estrategias y a veces, proporcionado herramientas para presionar a las instituciones y gobiernos en el cumplimiento de los objetivos anunciados (Milman y Short 2008; Hezri y Dovers 2006).

Sin embargo, estos modelos no están pensados para medir la capacidad de adaptación de los sistemas a los cambios y de mantener la funcionalidad en el tiempo. En general, la Sostenibilidad se concibe a partir de la definición de un estado estacionario, donde las condiciones y los recursos son estables en el tiempo. Por eso, los modelos existentes deben modificarse para incorporar la evaluación de la resistencia frente a las perturbaciones, la adaptabilidad y la capacidad de recuperación después de las crisis. En otras palabras, los modelos actuales deben incorporar el concepto de resiliencia entre los objetivos de la evaluación.

\subsection{I ndicadores de sostenibilidad urbana CAT-MED}

Como base para realizar este trabajo, se usa el sistema de indicadores de sostenibilidad CATMED, desarrollado a través de un proyecto de investigación Europeo del programa del "Cambio Climático y los riesgos naturales asociados". El proyecto ha contado con la participación de numerosas ciudades en los diferentes países de Europa, poniendo de manifiesto la relación entre Cambio Climático y desarrollo sostenible de las ciudades, con énfasis al área del Mediterráneo como una de las más afectadas por la subida del nivel del mar, la desertificación y las inundaciones. Las hipótesis de proyecto se basan en el reconocimiento de la relación existente entre la organización de la estructura urbana y el consumo de recursos, entendiendo las ciudades europeas (ciudad media mediterránea) como una estructura morfológica consolidada contrapuesta a la dispersión urbana, un modelo que ha aumentado el desequilibrio entre espacio construido y el entorno (Marín Cots 2012; Turégano Romero 2009).

La propuesta de actuación sobre el modelo urbano se organiza alrededor de tres conceptos claves: la complejidad, la compacidad y la proximidad a los servicios básicos. Estos conceptos relativos a la configuración urbana establecen unas pautas sobre las cuales encaminar el desarrollo de las ciudades hacía la sostenibilidad.

La compacidad es el parámetro que atiende a la realidad física, estando directamente relacionada con la densidad edificatoria, el uso de suelo, la cantidad de verde y de viario existente. Con este parámetro se quiere evaluar la proximidad entre usos y funciones urbanas, viendo en conjunto la cantidad de construido con la previsión de equipamiento y espacios públicos.

La complejidad atiende a la organización urbana, a la mezcla de usos y funciones en el territorio. La complejidad urbana refleja las interacciones que se establecen en el espacio urbano y que son espejo de la vitalidad de la ciudad. Este parámetro está ligado al concepto de diversidad urbana, muestra la madurez del tejido urbano y la riqueza del capital económico, social y biológico. 
El concepto de proximidad en el espacio urbano es expresión de la vecindad de la ciudad, afectando tanto a la generación de movilidad como a la cohesión social de los ciudadanos. Fariña (2009) define la proximidad como aquello que en el entorno urbano tiene un carácter doméstico, cercano a la vivienda, distribuido por la trama urbana, multifuncional. Un diseño urbano "de proximidad" promueve una gestión diferente del espacio, la movilidad peatonal, los comercios locales, el contacto con las personas y la cercanía de equipamientos y funciones.

Los indicadores desarrollados por CAT-MED están orientados a la definición de una ciudad "a la escala del hombre" como modelo del desarrollo urbano sostenible. Este estándar se basa principalmente en la estructura urbana, en su forma y su organización como medidas concretas para enfrentar los retos de la adaptación de las ciudades al Cambio Climático a la vez que proporciona a sus ciudadanos soluciones novedosas, eficientes y de calidad.

Para ello, se proponen una serie de indicadores organizados en 4 ejes de principales: Territorio y configuración de la ciudad, Movilidad y Transporte, Gestión de Recursos Naturales y Cohesión social y económica. Los indicadores están pensados como una herramienta de trabajo para el diseño y monitorización de los procesos de transformación urbana. Por tanto, se han propuesto 20 indicadores cuantitativos a los que se les asocia un método de cálculo y un valor o rango evaluación (Ver Tabla 1).

En este documento no se entrará en detalle a revisar los métodos de cálculo y rangos de evaluación puesto que no es su objetivo, sin embargo, se propone un acercamiento a la cuestión definiendo un marco teórico para la integración de los conceptos de resiliencia y de sostenibilidad urbana.

\subsection{El concepto de Resiliencia aplicado al entorno urbano}

Los recientes estudios sobre "la ciencias de la sostenibilidad" (Panel Intergubernamental sobre el Cambio Climático-IPCC, Consejo Internacional de Ciencias, Programas sobre el Cambio Ecológico y Social-PECS y Millennium Ecosystem Assessment) muestran un creciente interés en las nociones de resistencia al riesgo, vulnerabilidad y resiliencia (Walker et al. 2004; Turner 2010). Esto demuestra la necesidad de que la sostenibilidad evolucione incluyendo estos nuevos paradigmas para su desarrollo. Sin embargo, no existe un acuerdo entre los expertos sobre la definición de la resiliencia frente a los desastres y de la integración de este concepto en el desarrollo sostenible (Cutter, Ash y Emrich 2014; Jabareen 2012)

La resiliencia en el entorno urbano nace del estudio de como las formas en que los sistemas ecológicos hacen frente a las perturbaciones y cambios producidos por los factores externos. De acuerdo al concepto de sistema ecológico-social, podemos definir la resiliencia como "la capacidad de los sistemas: ciudades, comunidades o sociedades expuestas a amenazas de resistir, absorber, adaptarse o recuperarse de los efectos de las amenazas eficientemente y en un tiempo razonable, incluido el mantenimiento y recuperación de sus estructuras básicas o funciones" (J abareen, 2013:221).

Las diferentes teorías sobre el tema coinciden en la naturaleza compleja y multidisciplinar de la resiliencia urbana. Eso se debe a que la ciudad en si es un fenómeno complejo y multidisciplinar, en el cual es necesario un acercamiento holístico para la compresión real de sus procesos y de las interacciones entre ellos. Otro vacío en la literatura se debe al desconocimiento de cómo se puede evaluar la resiliencia de los sistemas específicos y del conjunto urbano en general (J abareen 2012).

De acuerdo al acercamiento de Holling (1973), la resiliencia se puede medir como la capacidad de los sistemas de pasar de un estado al siguiente manteniendo sus estructuras y funcionalidades básicas. Holling trata la resiliencia como un fenómeno dinámico donde los 
sistemas funcionan en base a series de ciclos de estabilidad aptándose a las diferentes perturbaciones y reorganizándose en nuevas configuraciones (Holling 2001; Walker et al. 2004). El concepto de tiempo y de imprevisibilidad de los eventos son entonces elementos claves para el estudio de la resiliencia y adaptabilidad de las ciudades.

Susan Cutter (2003), en sus estudios sobre la vulnerabilidad, relaciona la resiliencia de los sistemas socio-ecológicos con las condiciones geográficas. Sus investigaciones se basan en la hipótesis de que es posible asociar la vulnerabilidad a patrones espaciales y que una vez identificados estos conformen las directrices de la adaptabilidad del sistema urbano. El extenso listado de indicadores de vulnerabilidad propuesto por la autora supone un gran avance en la definición de un marco de estudio del entorno urbano. De acuerdo a los estudios publicados en la resiliencia se distinguen dos calidades: la inherencia (mantenimiento de las funcionalidades durante las crisis) y la flexibilidad (adaptación y respuesta durante el evento). Estas características son aplicables tanto a las infraestructuras, como a la organización institucional o a los sistemas sociales y económicos (Cutter, S. L., Boruff, B. J. and Shirley 2003; Cutter, Ash y Emrich 2014).

Una estructura urbana sostenible puede contribuir a la resiliencia en la medida en la que es capaz de resistir y mitigar el impacto de los eventos y, ser flexible a los cambios; aprovechando el trabajo en red y la capacidad de reorganizar las estructuras y recursos disponibles. Para ello, los modelos existentes deben considerar los factores de riesgo y la imprevisibilidad de los eventos, la interacción entre niveles y dimensiones. De esta manera se generarán herramientas que además de evaluar el estado del sistema podrán avisar sobre los aspectos críticos, ayudando en la toma de decisiones de acciones preventivas (Milman y Short 2008).

\section{Análisis de los indicadores}

Para la definición del marco teórico de referencia se analizan los indicadores de sostenibilidad de CAT-MED indicando para cada uno su contribución a la resiliencia urbana de acuerdo a la literatura en el tema.

\subsection{Territorio y configuración de la ciudad}

Estos indicadores se enfocan en la descripción de la morfología urbana. Sin bajar al detalle de los rangos de evaluación se puede observar que la estructura física del contexto urbano es determinante tanto la sostenibilidad como la resiliencia. Elementos como la complejidad, la variedad de uso y la compacidad son valiosos para ambos criterios.

En cuanto a la densidad urbana y al espacio verde, son factores claves en los dos sistemas aunque, es importante hacer una distinción en la función que tienen en los dos enfoques. En la sostenibilidad la densidad urbana es un indicador de compacidad, a mayor densidad menor el consumo de suelo. La presencia de verde en la ciudad es indicador de calidad del ambiente urbano, sirve como sumidero de polución, ayuda a reducir el impacto del ambiente construido y proporciona área de esparcimiento para los ciudadanos (Marín Cots 2012; Fariña Tojo 2007). En el caso de la resiliencia, tanto la densidad urbana como la provisión de verde deben ser analizados en conjunto y de acuerdo con la identificación de las áreas de riesgo. En la densidad urbana el factor determinante es la localización de población en zonas de riesgo. Según Cutter (2003) la densidad de construcción provee una información sobre lo que se podría perder por efecto del evento (Cutter, S. L., Boruff, B. J. and Shirley 2003). En la práctica, en los planes de reconstrucción post-tsunami en el 2010 en la localidad de Dichato y del borde fluvial de Constitución, se expropiaron los terrenos localizados en la primera línea de costa para la realización de las medidas de mitigación. Esta estrategia abrió un largo debate sobre si el 
desplazamiento de la población fuera del área de riesgo es beneficiosa o contraproducente, puesto que rompe los lazos culturales y comunitarios de aquellos que siempre vivieron allí (MINVU 2013). En los estudios realizados por Allan (2011) y Villagra (2013) del espacio libre se indica que juega un rol importante durante la fase de recuperación post-desastre. En el caso del terremoto de San Francisco en el 1960 y de Concepción en el 2010 los espacios públicos (parques, jardines, plazas, calles, etc.) fueron rápidamente ocupados por la población para organizar campamentos provisionales y actividades de emergencia (Villagra Isla y Rojas Quezada 2013; Allan y Bryant 2011).

En relación a la complejidad, es posible decir que los sistemas biológicos complejos (diversos en cuanto a especies y funciones) tienen mayor capacidad de reorganizarse después de una crisis. En analogía con los sistemas biológicos, también en las ciudades la complejidad es un elemento positivo para la resiliencia. La diversidad de ideas, conocimientos, organizaciones, culturas, etc. promovidas por los movimientos migratorios, por ejemplo, así como la diversidad en la estructura económica y productiva, puede ser el motor para la innovación (Walker et al. 2015).

\subsection{Movilidad y Transporte}

La movilidad es uno de los ejes claves de la sostenibilidad urbana. Las actuaciones en este campo están orientadas hacia la reducción de las emisiones de contaminantes, promoviendo sistemas de transporte alternativos, de bajo impacto ambiental, y amigables con el entorno. En presencia de riesgo de desastre, el transporte tiene una influencia directa e indirecta con la resiliencia. La directa se debe a la capacidad de movilidad de las personas después del evento, y está relacionada a los problemas que se pueden generar por la escasez de suministro de carburante o el colapso de las infraestructuras. La influencia indirecta se da a partir de los hábitos de desplazamiento de la población; la diversidad e intermodalidad mejoran la conectividad y redundancia. La conectividad a todos los niveles, dentro del sistema y entre el sistema (ciudad) y su entorno (espacio rural) es esencial para la recuperación. Por lo tanto, es necesario asegurar suministros básicos a la región sin depender del exterior, ser redundantes en las infraestructuras y promover la conectividad entre los ciudadanos y los lugares donde se organiza la emergencia. También es interesante asegurar una buena transmisión de la información, involucrando tanto los aspectos sociales como a las infraestructuras (Walker et al. 2015; Pickett y Zhou 2015). Una comunidad activa y vital, con una adecuada conectividad y un buen reparto modal es más resistente y tiene una mejor capacidad de recuperación después de una crisis.

Un aspecto muy importante a tener en cuenta en las áreas expuestas a riesgo es el problema de la evacuación. Para las comunidades expuestas a riesgos es fundamental poder asegurar una correcta y rápida evacuación de las personas hacía los espacios seguros. Las investigaciones realizadas por Murakami (2012) muestran los problemas relacionados con la evacuación de la costa de Yuriage durante el tsunami del 2011 en la costa este de Japón. Uno de los principales problemas que se verificó fue la evacuación con vehículos privados de un importante porcentaje de la población, esto provocó la congestión de las vías de comunicación y muchos no pudieron alcanzar los lugares seguros a tiempo, acarreando la perdida de muchas vidas humanas (Murakami, Takimoto y Pomonis 2012).

\subsection{Gestión de Recursos Naturales}

La categoría de indicadores relacionados con el uso de recursos es la que presenta mayor discrepancias entre los dos enfoques. La sostenibilidad se centra en la reducción del consumo y en la gestión eficiente de las fuentes renovables. En la resiliencia son interesantes otros aspectos relacionados con el funcionamiento en red (Colucci 2012), el mantenimiento de los servicios y el rol de las energías renovables en cuanto a la posibilidad de asegurar el 
mantenimiento de suministros básicos. Varios autores ponen énfasis en la necesidad de diseñar de forma integrada las infraestructuras atendiendo a las necesidades de adaptabilidad y mitigación (McCormick et al. 2013; The World Bank 2013).

Otros aspectos de la resiliencia son las reservas biológicas y socio-económicas útiles tras producirse el evento. En el caso del terremoto del 2010 en Chile, la población de Concepción y San Pedro de la Paz pudieron recolectar agua desde las lagunas y fuentes naturales existentes en el aglomerado urbano, permitiendo la presencia de estos elementos asegurar el suministro mínimo hasta la llegada de los servicios de emergencia (Allan et al. 2013). En el caso de Kobe, después del terremoto del 1995 la población impulsó la realización de nuevos parques urbanos pensados para la fase de emergencia, estos se dotaron de fuentes de agua potable, de lagunas que pueden transformarse en letrinas, de elementos que pueden servir de cocina y de árboles frutales en lugar de los decorativos (Bryant y Allan 2013).

\subsection{Cohesión social y económica}

El objetivo de la sostenibilidad es mantener el equilibrio entre los pilares ambiental, social y económico, por eso los indicadores que pertenecen a esta categoría están enfocados en garantizar el acceso equitativo a los servicios y a la vivienda digna (Marín Cots 2012; Naredo 2003). En el acercamiento a los indicadores socio-económicos de la resiliencia estos dan información sobre el nivel de vulnerabilidad y a la capacidad de recuperación de la comunidad después de una crisis.

Desde el punto de vista de la sostenibilidad los primeros tres indicadores de esta categoría entregan información sobre las condiciones de acceso a servicios, viviendas y empleo en la ciudad. Estos tienen una doble función, por un lado identificar los barrios que presentan una mayor degradación y por otro, medir el grado de compromiso de las instituciones públicas (Marín Cots 2012; Fariña Tojo 2009). En la ciudad resiliente esta información sirve para determinar el nivel de vulnerabilidad de la población (Cutter 1996) y para asegurar el acceso a servicios y equipamientos útiles durante la emergencia y la reconstrucción. Por ejemplo, en el acceso a los servicios básicos en la sostenibilidad nos interesa que haya variedad en los tipos de servicios proporcionados y que esos sean accesibles a todos los ciudadanos en un entorno de proximidad (Marín Cots 2012). Para la resiliencia el interés se enfoca a la distribución homogénea de los equipamientos de proximidad que pueden ser empleados para la organización de las actividades de emergencia (Villagra et al. 2014; Project 2011).

La cantidad de viviendas sociales no es muy relevante para la resiliencia di per se, sin embargo, permite asegurar un nivel mínimo de calidad y resistencia de las edificaciones en aquello lugares donde el porcentaje de informalidad es elevado (Cutter 1996; The World Bank 2013). Principalmente parece más interesante asegurar la capacidad de las administraciones locales en proveer soluciones habitacionales después del evento a través la financiación a la reconstrucción. Por eso, los gobiernos, además de proveer viviendas sociales de calidad y que incorporen medidas de mitigación adecuadas, deberían comprometerse en generar mecanismo que permitan la financiación de la reconstrucción, considerando soluciones como fondos de emergencia, externalización del riesgo y participación del sector privado y de los fondos internacionales (GFDRR 2015).

El turismo a pesar de ser un motor importante para las economías locales es una de las actividades que mayormente se ven afectadas por los desastres, por ello puede transformarse en un factor de vulnerabilidad en presencia de riesgo. En general se puede observar que las ciudades monofuncionales son más vulnerables frente al riesgo, la diversificación de los sectores económicos promueve que no haya colapso simultáneo de todas las actividades por efecto de la crisis (Walker et al. 2015). Cutter en sus indicadores identifica como factor de 
vulnerabilidad la dependencia del sector primario (agrícola, ganadero y pesquero) (Cutter, $\mathrm{S}$. L., Boruff, B. J. and Shirley 2003), sin embargo, se puede entender que la presencia de actividades de producción en la proximidad de las áreas afectadas pueden ser fuente de alimento en el caso de corte de suministro de provisiones después del desastre.

El último indicador está relacionado con la educación en los colegios el cual es un elemento muy importante en la resiliencia. Una buena educación sobre el riesgo, la preparación de la población a enfrentar la emergencia, el conocimiento de cómo realizar una correcta evacuación y de cómo actuar frente al riesgo es una de las medidas más efectivas para prevenir pérdidas humanas por efecto de los eventos naturales (Murakami, Takimoto y Pomonis 2012; The World Bank 2013).

\subsection{Indicadores Socio-Económicos Complementarios}

Estos indicadores son definidos como "complementarios" porque no están directamente relacionados con el diseño de la ciudad, pero proporcionan información necesaria para hacer un diagnóstico del área de estudio. En la ciudad sostenible la equidad social es uno de los objetivos esperados, al igual de los valores ambientales y la eficiencia económica (Naredo 2003; Fariña Tojo 2003). Para las comunidades afectadas por el riesgo, los indicadores socioeconómicos asumen un papel fundamental en la determinación del grado de vulnerabilidad y en la capacidad de recuperación post-desastre (Cutter 1996). Por ejemplo, Japón, un país que posee una gran capacidad de aprender del pasado durante el terremoto de 2011 las instituciones financieras pudieron mantener una operatividad estable durante la emergencia. Esto se debió a una efectiva cooperación entre las entidades privadas y pública que permitió utilizar recursos para la emergencia y reconstrucción inmediatamente después del desastre (GFDRR 2015).

\section{Resultados de la comparativa entre indicadores de sostenibilidad y de resiliencia}

A continuación se presentan los resultados de la evaluación de los indicadores de sostenibilidad de acuerdo a los principios de resiliencia. Se establece una escala según un patrón de colores que indica la relevancia de cada indicador de sostenibilidad en la resiliencia urbana. Esta valoración permite avanzar una discusión sobre las sinergias entre las estrategias, ver los solapes y diferencias.

En la gráfica se muestra la valoración del modelo de indicadores de sostenibilidad (Verde) y su contribución en la resiliencia (Azul). Para la valoración de la sostenibilidad se asocia a cada indicador una puntuación igual al valor máximo (Muy relevante=3) porque cada indicador es pertinente al diseño sostenible y un valor igual a 2 (Relevante) para los indicadores complementarios porque no están directamente relacionados con el diseño. En la columna de Resiliencia (ver Tabla 1) se muestra la valoración asignada a cada indicador como valoración de su contribución a la construcción de ciudades resilientes frente a los desastres de acuerdo al análisis presentado anteriormente. En la primera gráfica (Fig. 1.a) se presenta la evaluación de las primeras 4 categorías de indicadores y en la segunda gráfica (Fig. 1.b) se presenta la evaluación en las 5 categorías normalizando la categoría de los "Indicadores Socio-Económicos Complementarios" a una valoración de 0 a 15 para dar una representación homogénea del modelo en su conjunto.

En la primera gráfica (Fig. 1.a) podemos apreciar que el área de solape entre los dos conceptos es principalmente en los aspectos de diseño de "Territorio y configuración de la ciudad" y "Cohesión social y económica", mientras que las otras dos categorías "Movilidad y Transporte" y de "Gestión de los Recursos" tienen pocas sinergias entre los aspectos evaluados. Al añadir la categoría de los "Indicadores Socio-Económicos Complementarios" la gráfica muestra que en la 
resiliencia urbana los aspectos socios-económicos representan un elemento fundamental. El área de solape entre los dos enfoques aumenta enfatizando las sinergias en aquellos aspectos que atiende a la cohesión social y a la equidad económica.

Tabla 1 Resumen de los indicadores de sostenibilidad, descripción, diferencias y sinergias entre la sostenibilidad y la resiliencia. Las últimas dos columnas proponen la evaluación de la sostenibilidad (S) y de la resiliencia ( $R$ ) evaluada según una puntuación de 0 a 3.

\begin{tabular}{|c|c|c|c|c|c|}
\hline & INDICADOR & Diferencias & $\begin{array}{c}\text { Sinergias } \\
\end{array}$ & $\mathrm{S}$ & $\mathbf{R}$ \\
\hline \multirow{5}{*}{ 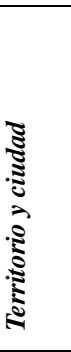 } & Densidad de población & $\begin{array}{l}\text { Alta densidad aumenta el riesgo de pérdidas } \\
\text { humanas y materiales. }\end{array}$ & $\begin{array}{l}\text { Baja densidad aumenta la dispersión de la } \\
\text { población en el territorio. }\end{array}$ & 3 & 2 \\
\hline & Compacidad urbana & Debe ser proporcional a la densidad de población. & $\begin{array}{l}\text { Es un recurso útil para la organización de la } \\
\text { emergencia. }\end{array}$ & 3 & 3 \\
\hline & Complejidad urbana & & $\begin{array}{l}\text { La diversidad es un elemento clave para la } \\
\text { resiliencia del sistema. }\end{array}$ & 3 & 3 \\
\hline & Zonas verdes & & $\begin{array}{l}\text { Es un recurso útil para la organización de la } \\
\text { emergencia. }\end{array}$ & 3 & 3 \\
\hline & Proximidad a zonas verdes & & $\begin{array}{l}\text { Es un recurso útil para la organización de la } \\
\text { emergencia. }\end{array}$ & 3 & 3 \\
\hline \multirow{5}{*}{ 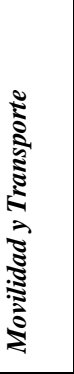 } & Reparto modal de tráfico & No es directamente relevante con la resiliencia. & $\begin{array}{l}\text { Un buen reparto modal es sinónimo de } \\
\text { diversidad y redundancia. }\end{array}$ & 3 & 1 \\
\hline & $\begin{array}{l}\text { Proximidad a paradas de } \\
\text { transporte público }\end{array}$ & No es relevante en un escenario de desastre. & & 3 & 0 \\
\hline & $\begin{array}{l}\text { Proximidad a la red de } \\
\text { carriles bicicleta }\end{array}$ & $\begin{array}{l}\text { No es directamente relacionado con una mayor } \\
\text { resiliencia frente a los desastre. }\end{array}$ & $\begin{array}{l}\text { Promueve una mayor conectividad útil en caso } \\
\text { de colapso de las conexiones. }\end{array}$ & 3 & 1 \\
\hline & $\begin{array}{l}\text { Porcentaje de calles y } \\
\text { espacios peatonales }\end{array}$ & & $\begin{array}{l}\text { Promueve una mayor conectividad útil en caso } \\
\text { de colapso de las conexiones }\end{array}$ & 3 & 2 \\
\hline & Emisiones de CO2 & No es directamente relevante con la resiliencia. & Preservar el capital ecológico & 3 & 1 \\
\hline \multirow{5}{*}{ 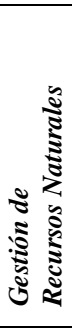 } & Consumo de energía & $\begin{array}{l}\text { Redundancia de la infraestructura, el funcionamiento } \\
\text { en red y el uso de energía renovable. }\end{array}$ & Preservar el capital ecológico & 3 & 0 \\
\hline & Consumo de agua & $\begin{array}{l}\text { Redundancia de la infraestructura, el funcionamiento } \\
\text { en red y el uso de fuentes locales. }\end{array}$ & Preservar el capital ecológico & 3 & 0 \\
\hline & $\begin{array}{l}\text { Gestión y recogida de } \\
\text { residuos }\end{array}$ & $\begin{array}{l}\text { Recogida y disposición de residuos evitando } \\
\text { problemas higiénicos. }\end{array}$ & $\begin{array}{l}\text { Fomentar el reciclado de los residuos a nivel } \\
\text { local. }\end{array}$ & 3 & 1 \\
\hline & Calidad de aire & $\begin{array}{l}\text { No es un factor directamente relacionado con la } \\
\text { resiliencia. }\end{array}$ & Riesgos para la salud de las personas. & 3 & 0 \\
\hline & Calidad del silencio & $\begin{array}{l}\text { No es un factor directamente relacionado con la } \\
\text { resiliencia. }\end{array}$ & Riesgos para la salud de las personas. & 3 & 0 \\
\hline \multirow{5}{*}{ 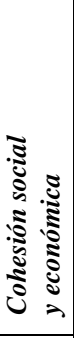 } & $\begin{array}{l}\text { Proximidad a servicios } \\
\text { básicos }\end{array}$ & & $\begin{array}{l}\text { Son espacios útiles para dar refugio y } \\
\text { desarrollar las actividades de emergencia. }\end{array}$ & 3 & 3 \\
\hline & $\begin{array}{l}\text { Porcentaje de viviendas } \\
\text { social }\end{array}$ & Conocer el estado de las viviendas. & $\begin{array}{l}\text { El manejo de la vulnerabilidad habitacional } \\
\text { aumenta la capacidad de recuperación. }\end{array}$ & 3 & 2 \\
\hline & \begin{tabular}{|l|} 
Tasa de población activa y \\
desempleo
\end{tabular} & $\begin{array}{l}\text { Es necesario tener información sobre la } \\
\text { vulnerabilidad económica }\end{array}$ & $\begin{array}{l}\text { El manejo de la vulnerabilidad económica } \\
\text { aumenta la capacidad de recuperación. }\end{array}$ & 3 & 3 \\
\hline & \begin{tabular}{|l|} 
Frecuencia turística \\
\end{tabular} & Puede ser factor de vulnerabilidad & Puede promover la recuperación. & 3 & 1 \\
\hline & Educación ambiental & Enfocar la educación a la gestión del riesgo. & $\begin{array}{l}\text { La educación aumenta el capital cultural de las } \\
\text { comunidades. }\end{array}$ & 3 & 2 \\
\hline \multirow{3}{*}{ 总 } & Renta media por hogar & & Nivel de vulnerabilidad de la comunidad. & 2 & 3 \\
\hline & Tasa de pobreza & & Nivel de vulnerabilidad de la comunidad. & 2 & 3 \\
\hline & Desigualdad de renta & & Nivel de vulnerabilidad de la comunidad. & 2 & \\
\hline
\end{tabular}

Fuente: CAT-MED y elaboración propia. 
Revista de Urbanismo N³4 - Enero - Junio de 2016 Departamento de Urbanismo - FAU - Universidad de Chile
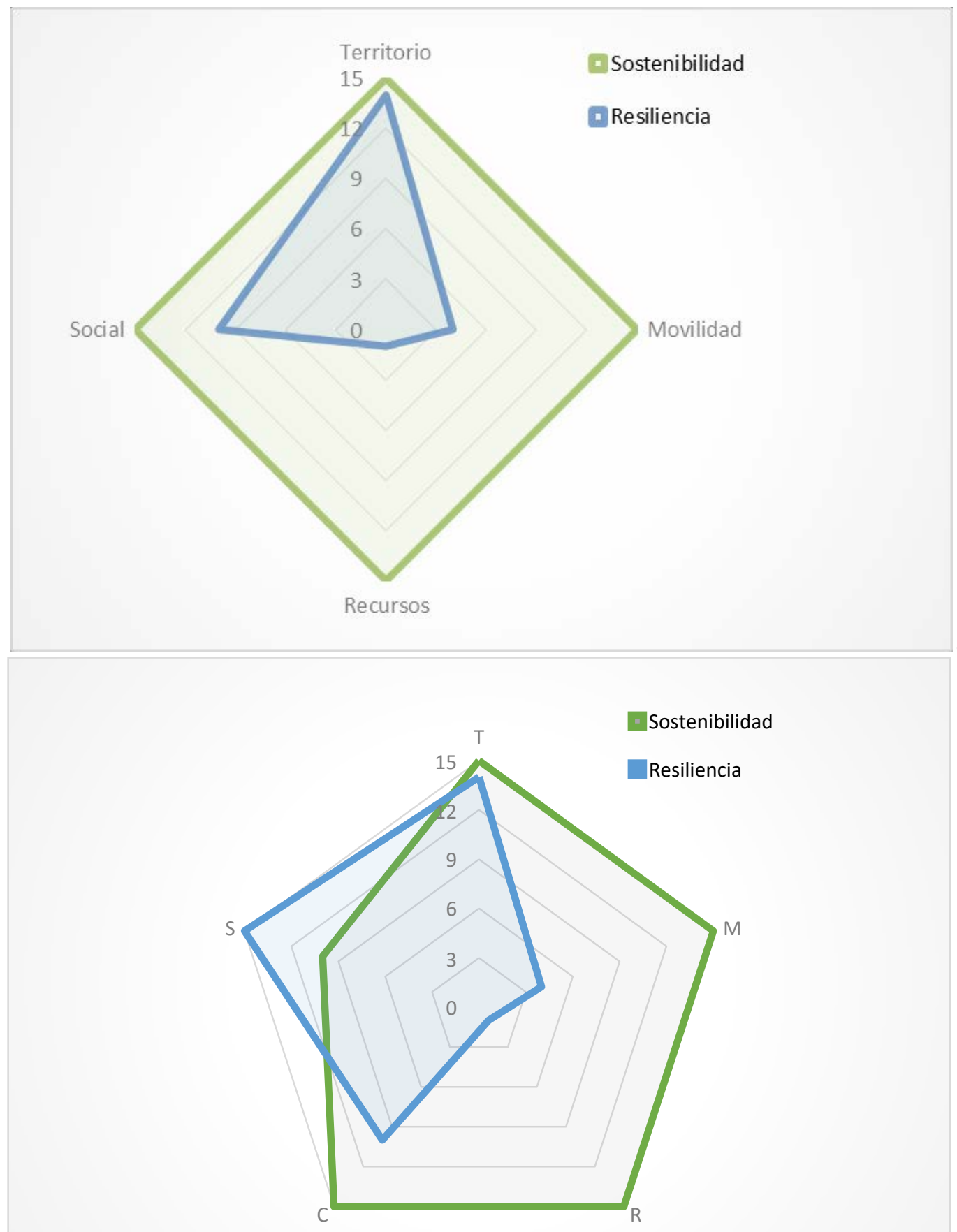

Fig. 1 Grafica comparativa entre sostenibilidad y resiliencia a) considerando las 4 categorías de indicadores, b) considerando las 4 categorías de indicadores y los indicadores complementarios. En verde la evaluación de la sostenibilidad y en azul el de la resiliencia

Fuente: Elaborado por el autor. 


\section{Discusión}

Los resultados obtenidos muestran que entre la sostenibilidad y la resiliencia existen solapes y similitudes que pueden favorablemente aprovecharse como sinergias para el desarrollo de las ciudades. En el análisis se aprecia una correspondencia entre los dos acercamientos en las categorías de "Territorio y configuración de la ciudad" y "Cohesión social y económica". Los indicadores de sostenibilidad son apropiados también para el diseño resiliente, aunque se destaca la necesidad de reorientar el enfoque de evaluación y ajustado los rangos de referencia. En el caso de la densidad urbana y de la compacidad estas deben relacionarse con las áreas de riesgo, o para la provisión de equipamientos, la evaluación debe orientarse a los objetivos de modularidad y del uso durante la emergencia. Si los indicadores de sostenibilidad se enfocan en asegurar un nivel adecuado de equipamiento para la integración de la resiliencia es necesario evaluar además su oportuna localización en el territorio. Los equipamientos son lugares útiles para las actividades de emergencias, por eso deben ser accesibles y adaptables a los cambios, como por ejemplo servir de alojamiento temporal después de un desastre natural (Villagra et al. 2014; Project 2011; Allan y Bryant 2011).

Sostenibilidad y Resiliencia pueden trabajar de forma sinérgica para promover la cohesión social, la equidad y la formación del capital social. Una comunidad cohesionada y equilibrada es más resistente porque tiene mayor capacidad de generar lazos y redes de apoyo, elementos claves para la recuperación temprana post-desastre (Gallardo 2006). En el caso de la educación es fundamental además de la educación ambiental, el conocimiento sobre riesgo, de las medidas de prevención y de cómo actuar durante los eventos. Actividades de formación e información deberían realizarse de forma constante involucrando a toda la comunidad (The World Bank 2013). La cohesión social en cuanto capacidad de la sociedad en cooperar es uno de los indicadores fundamentales de la resiliencia, en ayudarse unos a los otros, asociándose y trabajando de forma colaborativa. La equidad en la distribución de la renta, la identidad, la tolerancia y compartir una visión común son elementos que deberían promoverse desde el diseño urbano (Walker et al. 2015).

Las categorías de "Movilidad y Transporte" y de "Gestión de los Recursos" presentan pocas analogías entre los dos enfoques. En el caso de la sostenibilidad, se tiende a organizar eficientemente el sistema teniendo como objetivo la reducción del consumo de recursos y de las emisiones de contaminantes y de residuos. En presencia de riesgo, la resistencia y la redundancia son características esenciales para el mantenimiento de la funcionalidad de las infraestructuras y de los sistemas. Eso se puede obtener enfatizando el funcionamiento en red y a veces, duplicando algunas estructuras para asegurar que el colapso de una no perjudique el funcionamiento de todo el sistema (Colucci 2012; Ahern 2011).

Los indicadores de sostenibilidad vinculados con la movilidad deberían complementarse con la evaluación de la capacidad de evacuación en situación de emergencia (Murakami, Takimoto y Pomonis 2012). La comunicación entre los sectores no es solamente elemento fundamental para la creación de un entorno vital y sostenible, sino que también permite el trabajo en red entre las diferentes áreas de la ciudad fomentado que los sectores más resilientes puedan dar soporte a los más vulnerables. Los indicadores de resiliencia deben entonces enfocarse para medir la conectividad entre los ciudadanos y los servicios básicos útiles durante la emergencia especialmente a través de recorridos peatonales (Ahern 2011; Murakami, Takimoto y Pomonis 2012).

Según los principios de la evaluación de los servicios ecosistémicos, los ecosistemas son indispensables para el bienestar de las personas y por eso, los instrumentos que regulan la interacción con el hombre deben enfocarse a la sostenibilidad y al menor impacto posible (Zakri y Watson 2005). En presencia de riesgo los ecosistemas juegan un papel clave en los espacios 
urbanos, activándose durante la emergencia y proveyendo recursos básicos para la recuperación (Allan y Bryant 2011). La degradación y empobrecimiento del capital ecológico existente atenta al bienestar de las personas y a su capacidad de recuperación (Folke 2006; Walker et al. 2015).

De acuerdo a lo anterior, el uso de los recursos renovables puede ser muy provechosos. Para la sostenibilidad es interesante limitar el agotamiento de recursos y las emisiones. Para las ciudades resilientes los recursos renovables contribuyen a la disminución del impacto del cambio climático (Jabareen 2013), y proveer abastecimiento básico en caso de colapso de los servicios. Puede ser este el caso de los sistemas de micro y mini generación de energía, que pueden asegurar un servicio mínimo a las viviendas. Por otro lado, se debe indicar que es necesaria la separación de los residuos sólidos urbanos, se proveyendo estrategias para la diferenciación y vertido de los residuos en lugares que no supongan riesgo de contaminación ambiental y/o problemas para las personas (Project 2011).

\section{Conclusiones}

El trabajo propone un acercamiento teórico a la resiliencia urbana, necesario para el diseño de estrategias para la transformación de la ciudad. A partir de la revisión de la literatura y de los objetivos propuestos por el Marco Sendai se analizan los indicadores de sostenibilidad urbana encontrando sinergias y diferencias entre los dos conceptos.

Las principales dificultades en la integración de la resiliencia en los instrumentos de sostenibilidad urbana están asociadas con la incertidumbre de las amenazas y de la dificultad de preverlas. Los instrumentos de evaluación de sostenibilidad trabajan con condiciones de partida conocidas, mientras que el desastre natural desplaza el sistema en una nueva condición, no predecible con anterioridad y que obliga al sistema a buscar nuevas estructuras y nuevas reorganizaciones (Jabareen 2013; Folke 2006). Sin embargo, también existen sinergias entre los dos conceptos en las cuales se pueden encontrar las claves para la definición de estrategias resilientes y a la vez sostenibles.

A través del análisis de los indicadores de sostenibilidad es posible resumir las claves para la integración de la resiliencia en los indicadores de sostenibilidad urbana como sigue:

- En cuanto a los indicadores de ocupación de suelo, es necesario introducir el estudio de las áreas de riesgo y definir las actividades y densidades en relación al grado de las amenazas.

- La movilidad debe sustituirse por la conectividad física y social entre los grupos que conforman el sistema y entre el sistema y su entorno, estableciendo redes que permitan la comunicación y el intercambio de recursos e información útil para la resiliencia (Ahern 2011; Childers et al. 2015; Pickett, Cadenasso y Grove 2004).

- Los recursos se deben evaluar de acuerdo a los servicios ecosistemicos que entregue la comunidad, considerándolos como recursos escasos e insustituibles, indispensables para la recuperación de la comunidad (Walker y Salt 2006; Folke 2006; Pickett et al. 2011).

- El diseño sostenible es fundamental para reducir las vulnerabilidades e inequidades de los espacios urbanos. Las comunidades más vulnerables son menos resistentes frente a los riesgos, por eso las acciones en el espacio urbano deben dirigirse a equilibrar las brechas existentes a nivel ambiental, social y económico (Cutter, S. L., Boruff, B. J. and Shirley 2003; Jabareen 2009).

- Añadir el concepto de incertidumbre a los instrumentos de planificación, entendiendo la perturbación como "oportunidad" y como espacio para "aprender haciendo". Los 
procesos de planificación deben ser flexibles, permitiendo que las políticas y estrategias propuestas puedan modificarse en un proceso continuo de experimentación y aprendizaje (Ahern 2011; J abareen 2009).

El análisis realizado se limita a estudiar la ciudad como sistema socio-ecológico, permitiendo de esta manera alcanzar conclusiones relevantes para la planificación urbana. Sin embargo, las relaciones existentes entre las diferentes escalas (local, territorial, regional-nacional) son determinantes para la resiliencia y necesitan de estudios más específicos para entender como las influencias mutuas afectan la capacidad de adaptación y recuperación de las comunidades. Este primer estudio permite avanzar en la identificación de las sinergias y diferencias entre los dos enfoques, conformando así una base teórica para el desarrollo de futuras investigaciones aplicadas en casos reales.

\section{Referencias}

Ahern, J. (2011). From fail-safe to safe-to-fail: Sustainability and resilience in the new urban world. Landscape and Urban Planning, 341-343. http://dx.doi.org/10.1016/j. landurbplan.2011.02.021

Allan, P. y Bryant, M. (2011). Resilience as a framework for urbanism and recovery. Journal of Landscape Architecture, 6(2), 34-45. https://doi.org/10.1080/18626033.2011.9723453

Allan, P., Bryant, M., Wirsching, C., García, D., \& Teresa Rodríguez, M. (2013). The influence of urban morphology on the resilience of cities following an earthquake. Journal of Urban Design, 18(2), 242-262. https://doi.org/10.1080/13574809.2013.772881

Benson, M. H. \& Craig, R. K. (2014). The end of sustainability. Society \& Natural Resources, 27(7), 777-782. https://doi.org/10.1080/08941920.2014.901467

Bryant, M. \& Allan, P. (2013). Open space innovation in earthquake affected cities. En J. Tiefenbacher (Ed.), Approaches to Disaster Management - Examining the Implications of Hazards, Emergencies and Disasters. https://doi.org/10.5772/55465

Cascio, J. (2009). The next big thing: Resilience. Foreign Policy 15, 11-12. Recuperado de http://www.openthefuture.com/media/FP-cascio.pdf

Childers, D., Cadenasso, M., Grove, J., Marshall, V., McGrath, B., \& Pickett, S. (2015). An ecology for cities: A transformational nexus of design and ecology to advance climate change resilience and urban sustainability. Sustainability, 7(4), 3774-3791. ISSN 2071-1050. https://doi.org/10.3390/su7043774

Colucci, A. (2012). Towards resilient city. Comparing approaches/strategies. TeMA, Journal of Land Use, Mobility and Environment, 5(2), 101-116. http://dx. doi.org/10.6092/1970-9870/921

Cutter, S. L., Boruff, B. J., \& Shirley, W. L. (2003). Social vulnerability to environmental hazards. Social Science Quarterly, 84(2), 242-261. https://doi.org/10.1111/1540-6237.8402002

Cutter, S. L. (1996). Vulnerability to environmental hazards. Progress in Human Geography, 20(4), 529-539. https://doi.org/10.1177/030913259602000407

Cutter, S. L., Ash, K. D., \& Emrich, C. T. (2014). The geographies of community disaster resilience. Global Environmental Change, 29, 65-77. https://doi.org/10.1016/j.gloenvcha.2014.08.005 
Fariña Tojo, J. (2003). Ciudades menos insostenibles. Boletín CF S, 25. Recuperado de http://habitat.aq.upm.es/boletin/n25/ajfar.html

Fariña Tojo, J. (2007). La ciudad y el medio natural. Madrid: Akal.

Fariña Tojo, J. (2009). Parques de proximidad en el siglo XXI. Recuperado de http://elblogdefarina.blogspot.cl/2009/03/parques-de-proximidad-en-elsiglo-xxi.html

Folke, C. (2006). Resilience: The emergence of a perspective for social-ecological systems analyses. Global Environmental Change, 16(3), 253-267. https://doi.org/10.1016/j.gloenvcha.2006.04.002

Gallardo, P. (2006). Espacio público como plataforma democratizante. El caso de la comuna de La Pintana. Recuperado de www.ucentral.cl/fid/trabajos_estud...10/patricia_gallardo.pdf

Global Facility for Disaster Reduction and Recovering, GFDRR. (2015). Resilient recovery: An imperative for sustainable development. Recuperado de http://www.preventionweb. net/files/44171 resilientrecoveryanimperativeforsus.pdf

Hezri, A. A. \& Dovers, S. R. (2006). Sustainability indicators, policy and governance: Issues for ecological economics. Ecological Economics, 60(1), 86-99. https://doi.org/10.1016/j.ecolecon.2005.11.019

Holling, C. S. (2001). Understanding the complexity of economic, ecological, and social systems. Ecosystems, 4(5), 390-405. https://doi.org/10.1007/s10021-001-0101-5

Jabareen, Y. (2012). Vulnerability of cities to extreme space weather events: A new frontier of a multidisciplinary urban research. Natural Science, 4(6), 368-371. https://doi.org/10.4236/ns.2012.46051

Jabareen, Y. (2013). Planning the resilient city: Concepts and strategies for coping with climate change and environmental risk. Cities, 31, 220-229. https://doi.org/10.1016/j.cities.2012.05.004

Jabareen, Y. (2009). Building a conceptual framework: philosophy, definitions, and procedure. International Journal of Qualitative Methods, 8(4), 49-62. https://doi.org/10.1177/160940690900800406

Marín Cots, P. (2012). Modelos urbanos sostenibles. Metodología de trabajo y resultados. Recuperado de http://www.catmed.eu/archivos/desc7_CatMed\%20Esp-Eng.pdf

Mccormick, K., Anderberg, S., Coenen, L., \& Neij, L. (2013). Advancing sustainable urban transformation. Journal of Cleaner Production, 50, 1-11. https://doi.org/10.1016/j.jclepro.2013.01.003

Milman, A. \& Short, A. (2008). Incorporating resilience into sustainability indicators: An example for the urban water sector. Global Environmental Change, 18(4), 758-767. https://doi.org/10.1016/j.gloenvcha.2008.08.002 
Ministerio de Vivienda y Urbanismo de Chile, Minvu. (2013). Reconstrucción Urbana post $27 \mathrm{~F}$. Instrumentos de planificación y gestión territorial. Recuperado de http://www.minvu.cl/incjs/download.aspx?glb_cod_nodo $=20131008125015 \&$ hdd_nom_archivo $=R$ econstruccion\%20urbana\%2027F.pdf

Murakami, H., Takimoto, K., \& Pomonis, A. (2012). Tsunami evacuation process and human loss distribution in the 2011 Great East Japan Earthquake, a case study of Natori City, Miyagi Prefecture. 15th World Conference on Earthquake Engineering. Recuperado de http://www.iitk.ac.in/nicee/wcee/article/WCEE2012_1587.pdf

Naredo, J. M. (2003). Instrumentos para paliar la insostenibilidad de los sistemas urbanos. Boletín CF S, 24. Recuperado de http://habitat.aq.upm.es/boletin/n24/ajnar.html

Pickett, S. T. A., Cadenasso, M. L., \& Grove, J. M. (2004). Resilient cities: Meaning, models, and metaphor for integrating the ecological, socio-economic, and planning realms. Landscape and Urban Planning, 69(4), 369-384. http://dx.doi.org/10.1016/j.landurbplan.2003.10.035

Pickett, S. T. A., Cadenasso, M. L., Grove, J. M., Boone, C. G., Groffman, P. M., Irwin, E., ... Warren, P. (2011). Urban ecological systems: Scientific foundations and a decade of progress. Journal of environmental management, 92(3), 331-362. https://doi.org/10.1016/j.jenvman.2010.08.022

Pickett, S. T. A. \& Zhou, W. (2015). Global urbanization as a shifting context for applying ecological science toward the sustainable city. Ecosystem Health and Sustainability, 1(1), art5-art5. https://doi.org/10.1890/ehs14-0014.1

Project, T. S. (2011). The sphere handbook: Humanitarian charter and minimum standards in humanitarian response. Recuperado de http://www.sphereproject.org/handbook/

The World Bank. (2013). Building urban resilience. https://doi.org/10.1596/978-0-8213-8865-5

Turégano Romero, J. A. (2009). Arquitectura bioclimática y urbanismo sostenible. Zaragoza: Publicaciones de la Universidad de Zaragoza.

Turner, B. L. (2010). Vulnerability and resilience: Coalescing or paralleling approaches for sustainability science? Global Environmental Change, 20(4), 570-576. http://dx.doi.org/10.1016/j.gloenvcha.2010.07.003

The United Nations Office for Disaster Risk Reduction, UNISDR. (2005). Summary of the Hyogo Framework for Action 2005-2015. Word Conference on Disaster Reduction. Kobe: Autor.

The United Nations Office for Disaster Risk Reduction, UNISDR. (2015). Marco de Sendai para la Reducción del Riesgo de Desastres 2015-2030. Recuperado de http://www2.ohchr.org/spanish/bodies/hrcouncil/docs/gaA.RES.60.1_Sp.pdf

Villagra Isla, P. y Rojas Quezada, C. (2013). Dimensiones física y cultural de la resiliencia posdesastre: ¿son compatibles en ciudades Chilenas? Geo Sur 4, 4(6), 85-102. 
Villagra, P., Rojas, C., Ohno, R., Xue, M., y Gómez, K. (2014). A GIS-base exploration of the relationships between open space systems and urban form for the adaptive capacity of cities after an earthquake: The cases of two Chilean cities. Applied Geography, 48, 64-78. http://dx.doi.org/10.1016/j.apgeog.2014.01.010.

Walker, B., Abel, N., Andreoni, F., Cape, J., Murdoch, H., Norman, ... White, R. (2015). General resilience. A discussion paper based on insights from a catchment management area workshop in southeastern Australia. Recuperado https://www.resalliance.org/files/General_Resilience_paper.pdf

Walker, B., Holling, C. S., Carpenter, S. R., \& Kinzig, A. (2004). Resilience, adaptability and transformability in social-ecological systems. Ecology and society, 9(2), 5. https://doi.org/10.5751/es-00650-090205

Walker, B. \& Salt, D. (2006). Resilience thinking: Sustaining ecosystems and people in a changing world. Washington: Autor.

Zakri, A. H. \& Watson, R. (2005). Ecosystems and Human Well-being. Ecosystems and Human Wellbeing: A Framework for Assessment. Recuperado de https://www. millenniumassessment.org/documents/document. 356.aspx.pdf 